\title{
El cambio climático desde un enfoque del derecho internacional de los derechos
}

bumanos

\section{Climate Change from an International Human Rights Law Approach}

Krúpskaya Rosa Luz Ugarte Boluarte* https://orcid.org/0000-0001-5226-4807

Lisbeth Orosco López** https://orcid.org/0000-0001-9365-4174

http://dx.doi.org/10.21503/lex.v19i28.2313

* Abogada. Doctora y Magister por la Universidad Carlos III de Madrid - España (CUMLAUDE). Especialista en el Área de Derecho Internacional de los Derechos Humanos. Directora Nacional de la Liga Peruana Pro Derechos Humanos (LIPPRODEH) y Cátedra Libre DIDH, consultora en Derechos Humanos, entre otros. Es Docente titular de la Cátedra de Derechos Humanos y Derecho Internacional Público de la Facultad de Derecho y Ciencias Políticas de la Universidad Nacional Mayor de San Marcos y en otras casas de estudio. Perú.

Correo electrónico: krupskaya74@hotmail.com

** Bachiller en Derecho de la Facultad de Derecho y Ciencias Políticas de la Universidad Nacional Mayor de San Marcos, cuenta con estudios en derecho internacional, derecho ambiental y cambio climático, llevó curso en la Escuela de Cambio Climático de la Universidad de Oxford, entre otras instituciones, como en la Escuela Superior del Personal del Sistema de las Naciones Unidas - Centro de Conocimiento para el Desarrollo Sostenible y Cambio Climático de la ONU, también es Miembro colaborador del Taller de Derecho Ambiental-UNMSM.

Correo electrónico: jlol.orosco.lopez@gmail.com

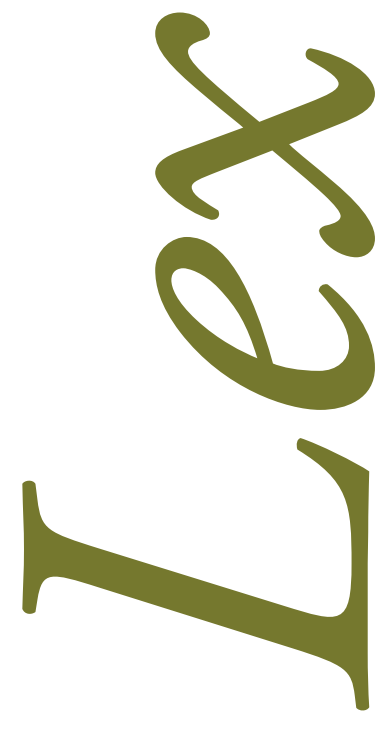




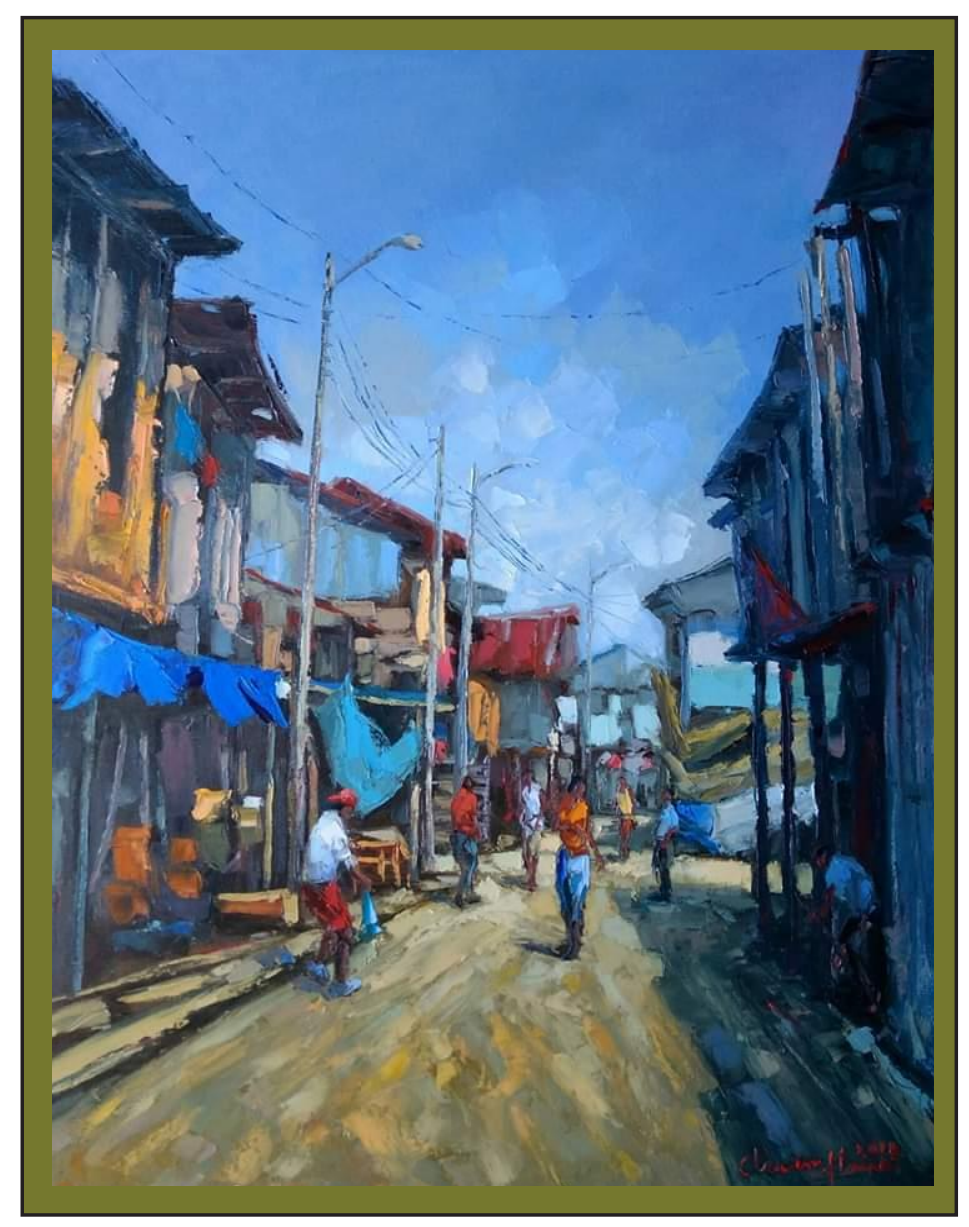

Belén zona baja. Óleo sobre lienzo, 81 x $100 \mathrm{~cm}$. Cliver Flores Lanza (Iquitos, Loreto, Perú, 1965) Correo electrónico: floreslanza@yahoo.com www.cliverpintoramazonico.blogspot.com 


\section{RESUMEN}

Este artículo describe los avances del desarrollo del Derecho Internacional Ambiental respecto al cambio climático y los impactos de este a los derechos humanos, en especial estando en un contexto internacional que demanda la acción climática global teniendo como base los últimos reportes científicos alarmantes. La aparición de litigios en materia de cambio climático continúa desarrollándose, mucho de los cuales reclaman la acción a gobiernos y a empresas cumplir con sus obligaciones, incluso alegando afectación a derechos humanos.

Palabras clave: Derecho Internacional Ambiental, cambio climático, derechos humanos.

\section{ABSTRACT}

This article describes the progress of the development of international environmental law with respect to climate change and its impacts on human rights, especially in an international context that demands global climate action based on the latest alarming scientific reports. The emergence of litigation on climate change continues to develop, many of which call for action by governments and companies to comply with their obligations, even alleging human rights impacts.

Key words: International Environmental Law, climate change, human rights. 


\section{INTRODUCCIÓN}

Hace más de cuatro décadas desde la adopción de la Declaración de Estocolmo, que se reconoció el inicio y desarrollo del Derecho Internacional Ambiental, donde se adoptaron reglas, normas, principios y tratados internacionales en materia ambiental. El cambio climático es uno de los temas más urgentes de la agenda global de los Estados, debido a los efectos que se producen alrededor del planeta. En específico, se desarrolló la regulación internacional sobre cambio climático con la Convención Marco de las Naciones Unidas sobre Cambio Climático, Protocolo de Kioto y el Acuerdo de Paris. Por otro lado, este fenómeno impacta directamente en los derechos humanos, ello causa preocupación y es motivo para realizar un análisis desde el derecho internacional de los derechos humanos, descansando en algunas instituciones del derecho internacional público. Este marco internacional de protección crea un orden legal, genera un marco teórico que va normar la conducta de los Estados. El objeto de estas normas, es lograr en definitiva la paz social, bienestar mundial inmersos en el desarrollo económico, etc. Es importante reconocer el Principio 25 de la Declaración de Río que señala: "La paz, el desarrollo y la protección del medio ambiente son interdependientes e inseparables". La disciplina del derecho ambiental internacional, está compuesto por el conjunto de normas y principios cuyo objeto es regular las conductas de los Estados y las Organizaciones internacionales, comprometiéndose de esta forma con el ambiente saludable y el desarrollo saludable en el marco del derecho internacional de los derechos humanos.

La Constitución Política del estado de 1993, en su artículo 2 inciso 22 señala que: "A la paz, a la tranquilidad, al disfrute del tiempo libre y al descanso, así como a gozar de un ambiente equilibrado y adecuado al desarrollo de su vida”. El artículo 67 señala: El Estado determina la política nacional del ambiente. Promueve el uso sostenible de sus recursos naturales, definiendo la política nacional del ambiente, cuyo objetivo es proteger los recursos naturales y el ambiente natural en que se desarrolla la vida humana. Entre los Derechos Humanos ambientales, regulados están: Artículo 11-derecho a un medio ambiente sano; Artículo 12- derecho a la vida; Artículo 13-derecho a la integridad personal; Artículo 14-derecho a la igualdad ambiental ; Artículo 15- derecho del consumidor; Artículo 16- derecho al desarrollo sostenible; Artículo 17 -derecho a acceder a información ambiental; Artículo 17 BIS..-derecho a la identidad ambiental, Artículo 18 -derecho a participar en decisiones que afecten al medio ambiente; Artículo 19-garantías judiciales 
El Perú es estado parte de los siguientes tratados: 1) Convención para la Protección de la Flora, de la Fauna y de las Bellezas Escénicas Naturales de los Países de América, 2) Convención para el Comercio Internacional de Especies Amenazadas de Extinción (CITES) ${ }^{1}$, 3) Acuerdo entre Perú y Brasil para la conservación de la flora y de la fauna de la Amazonía², 4) Tratado de Cooperación Amazónica, 5) Acuerdo entre Perú y Colombia para la conservación de la flora y de la fauna de la Amazonía ${ }^{3}$, 6) Convenio para la Conservación y Manejo de la Vicuña, 7) Convención para la protección del patrimonio mundial cultural y natural, 8) Convenio sobre Diversidad Biológica ${ }^{4}$, 9) Convención de Viena para la Protección de la Capa de Ozono, 10) Protocolo de Montreal sobre Sustancias que Agotan la Capa de Ozono $^{5}$, 11) Convenio de Basilea sobre el control de los movimientos transfronterizos de los desechos peligrosos y su eliminación, 12) Convención Marco de las Naciones Unidas sobre Cambio Climático ${ }^{6}$, 14) Convención Relativa a los humedales de importancia internacional especialmente como hábitat de aves acuáticas (RAMSAR, 1971), entre otros.

\section{DERECHO INTERNACIONAL DE LOS DERECHOS HUMANOS}

Hoy es importante enfatizar en la necesidad de tener un ambiente saludable, es uno de los derechos humanos de tercera generación, como la paz, el desarrollo y al patrimonio común, etc., llamados también derechos de solidaridad, este derecho comprender proteger el derecho a la vida, a la salud, que también son objeto de protección por otros tratados o instrumentos internacionales. Es una necesidad hablar del derecho humano a vivir en un ambiente mundial sano, solo así podremos gozar de los derechos económicos y políticos. Es un valor que permitirá la supervivencia humana y la biosfera. Se vinculan los siguientes tratados: 1) Declaración Universal de Derechos Humanos, 2) Declaración Americana de Derechos y Deberes del Hombre, 3) Pacto Internacional de Derechos Civiles y Políticos, 4) Pacto Internacional de Derechos Económicos, Sociales y Culturales, 5) Convención Americana sobre Derechos Humanos y 6) Carta de la Organización de Estados Americanos. Conforme podemos analizar:

1. Firmada en 1973 y ratificada por el Perú en 1974. Compromete a establecer controles de comercio de productos y especies de flora y fauna amenazadas de extinción.

2. Firmado en 1975 y que compromete a los dos países a cooperar en la conservación de la flora y fauna amazónicas.

3. Firmado en 1979. Establece un compromiso entre ambos países para cooperar en la conservación de la flora y fauna silvestres.

4. Firmado en Río de Janeiro en 1992 y ratificado en 1993, establece los compromisos mundiales y nacionales referentes a la identificación y monitoreo de la biodiversidad; la conservación in situ (áreas protegidas, introducción de especies foráneas, mantención y protección los conocimientos de las poblaciones locales)

5. Adoptada el 16 de setiembre de 1987. Establece normas para prohibir o limitar el uso de sustancias que afectan la estabilidad de la capa de ozono.

6. Adoptada el 4 de junio de 1992. Establece el marco internacional para encauzar acciones conjuntas para la prevención de los cambios climáticos a nivel global. 


\begin{tabular}{|c|c|}
\hline $\begin{array}{l}\text { Declaración Universal de Derechos } \\
\text { Humanos }\end{array}$ & $\begin{array}{l}\text { Artículo 3: "Todo individuo tiene derecho a la vida, }(\ldots) \text { ". } \\
\text { Artículo 25: "Toda persona tiene derecho a un nivel de vida adecuado } \\
\text { que le asegure la salud y bienestar". }\end{array}$ \\
\hline $\begin{array}{l}\text { Declaración Americana de Derechos y } \\
\text { Deberes del Hombre }\end{array}$ & $\begin{array}{l}\text { Artículo 1: "Todo ser humano tiene derecho a la vida, }(\ldots) \text { ". } \\
\text { Artículo 11: "Toda persona tiene derecho a que su salud sea } \\
\text { preservada" }\end{array}$ \\
\hline $\begin{array}{l}\text { Pacto Internacional de Derechos Civiles } \\
\text { y Político. }\end{array}$ & Artículo 6: "El derecho a la vida e \\
\hline $\begin{array}{l}\text { Pacto Internacional de Derechos } \\
\text { Económicos, Sociales y Cultural. }\end{array}$ & $\begin{array}{l}\text { Artículo 11: “...Derecho a una mejora continua de las condiciones de } \\
\text { existencia”. }\end{array}$ \\
\hline $\begin{array}{l}\text { Convención Americana sobre Derechos } \\
\text { Humanos. }\end{array}$ & Artículo 4: "Toda persona tiene derecho a que se le respete su vida". \\
\hline $\begin{array}{l}\text { Carta de la Organización de Estados } \\
\text { Americanos. }\end{array}$ & $\begin{array}{l}\text { Artículo } 44 \text { incisos a y b: "Derecho al bienestar material y a un trabajo } \\
\text { que permita asegurar la vida, la salud". }\end{array}$ \\
\hline
\end{tabular}

\section{Ilustración 1. Elaboración propia}

Todo este marco normativo internacional vincula el medio ambiente sano y los derechos humanos, para lograr un cambio climático que descanse en la conciencia mundial. Es importante precisar que existen Declaraciones sin fuerza ejecutiva en esta materia:

\begin{tabular}{|l|l|l|}
\hline Declaración de principios y & "Primer foro en el que la Comunidad mundial puso a discusión los \\
recomendaciones para la acción de la & $\begin{array}{l}\text { la } \\
\text { problemas ambientales y propuso soluciones. Principio I: "El } \\
\text { sonferencia de las Naciones el Medio Humano (Estocolmo 1972). } \\
\text { hombre tiene el derecho fundamental a la libertad, la igualdad y el } \\
\text { disfrute de condiciones de vida adecuadas en un medio de calidad } \\
\text { tal que le permita llevar una vida digna y gozar de bienestar y tiene } \\
\text { la solemne obligación de proteger y mejorar el medio para las } \\
\text { generaciones presentes y futuras". }\end{array}$ \\
\hline Carta Mundial de la Naturaleza (1982) & $\begin{array}{l}\text { "Parte del principio de que el derecho a la vida es esencial, que } \\
\text { debe ser la guía de las acciones humanas. Se desarrollaron 16 } \\
\text { principios, clasificados temáticamente en cuatro apartados:1) } \\
\text { Respeto y cuidado de la Comunidad de la vida, 2) Integridad } \\
\text { ecológica, 3) Justicia Social y económica y 4) Democracia, no } \\
\text { violencia y paz". }\end{array}$ \\
\hline
\end{tabular}




\begin{tabular}{|c|c|}
\hline Declaración sobre el Desarrollo (1987) & $\begin{array}{l}\text { "El derecho al desarrollo en alguna medida pretendió ser una } \\
\text { conquista de los países subdesarrollados". }\end{array}$ \\
\hline $\begin{array}{l}\text { Declaración de la Conferencia de las } \\
\text { Naciones Unidas sobre el Medio Ambiente } \\
\text { y Desarrollo (Río 1992). }\end{array}$ & $\begin{array}{l}\text { "Se consagra el desarrollo sostenible por reconociendo que es la } \\
\text { persona humana y no la naturaleza el centro de las preocupaciones } \\
\text { relacionadas con el desarrollo sostenible". }\end{array}$ \\
\hline Declaración de Viena (1993). & $\begin{array}{l}\text { "Establece el compromiso de realizar el desarrollo ajustado a la } \\
\text { protección ambiental. Se reconoce también el derecho que asiste a } \\
\text { las futuras generaciones de disfrutar de un ambiente sano". }\end{array}$ \\
\hline Cumbre Social (1995). & $\begin{array}{l}\text { "Plantea el gran reto de lograr el desarrollo económico junto con } \\
\text { el desarrollo sostenible, en ese sentido propone la necesidad de } \\
\text { lograr un entorno político y jurídico apropiado, en el que los } \\
\text { ciudadanos: Adquieran conciencia de sus responsabilidades para la } \\
\text { vida en sociedad". }\end{array}$ \\
\hline $\begin{array}{l}\text { Declaración del Milenio de } 2000 \text { y los } \\
\text { objetivos de Desarrollo del Milenio. }\end{array}$ & $\begin{array}{l}\text { "Fue aprobada en la Cumbre del Milenio llevada a cabo en } \\
\text { septiembre de } 2000 \mathrm{y} \text { ha sido firmada por los } 191 \text { Estados } \\
\text { miembros de la ONU. Contiene un conjunto de objetivos para } \\
\text { combatir la pobreza, el hambre, las enfermedades, el } \\
\text { analfabetismo, la degradación del ambiente y la discriminación } \\
\text { contra la mujer". }\end{array}$ \\
\hline $\begin{array}{l}\text { Cumbre Mundial sobre el Desarrollo } \\
\text { Sostenible (Johannesburgo 2002). }\end{array}$ & $\begin{array}{l}\text { "Los temas ambientales que pueden considerarse avances en } \\
\text { Johannesburgo son las siguientes:1) La valoración del agua potable } \\
\text { con la expectativa de que casi todos los seres humanos tengan } \\
\text { acceso a ella y a las tecnologías para el tratamiento de las aguas } \\
\text { residuales. 2) El propósito de dejar que se recuperen las existencias } \\
\text { de peces al 2015. 3) Reducir sensiblemente el uso de sustancias } \\
\text { químicas venenosas al 2020. 4) Reducir considerablemente la } \\
\text { pérdida de diversidad biológica, etc. }\end{array}$ \\
\hline
\end{tabular}

Tabla 1. Elaboración propia

Es importante que los Estados se comprometan con todos estos compromisos internacionales que contienen reglas y principios para regular las conductas de los sujetos de derecho internacional. Es tarea de los Estado proteger los ecosistemas como la Amazónica, el Chaco y la Puna entre otros. En ese 
sentido el trabajo de los Organismos internacionales y los Estados es la protección de la naturaleza y el desarrollo sostenible de los pueblos, y regular y resolver los problemas ambientales de alcance mundial como el cambio climático, agotamiento de la capa de ozono, erosión de la diversidad biológica, desertificación y crear las condiciones para el desarrollo sostenible.

\section{DERECHO INTERNACIONAL AMBIENTAL}

El hito internacional que marcó la pauta del Derecho Internacional Ambiental fue la adopción de la Declaración de Estocolmo en 1972, dicho instrumento marco una importancia significativa para los Estados que participaron en su adopción. Anglés, M., Rovalo, M. y Tejado, M , señalan que esta Declaración:

Reconoce por primera vez los principios internacionales en materia ambiental, documento jurídicamente no vinculante (soft law), pero que estableció un catálogo de principios en materia ambiental que se convertirían en una referencia obligada. Como resultado indirecto de la Conferencia fue la creación del Programa de Naciones Unidas para el Medio Ambiente (PNUMA) en 1972, con sede en Kenia, Nairobi.

Entre algunos de estos principios que describe la Declaración de Estocolmo, podemos mencionar: "Los recursos naturales de la Tierra, incluidos el aire, el agua, la tierra, la flora y la fauna y especialmente muestras representativas de los ecosistemas naturales, deben preservarse en beneficio de las generaciones presentes y futuras mediante una cuidadosa planificación u ordenación, según convenga”. (principio 2 de la Declaración de Estocolmo, 1972).

Posterior a Estocolmo, también marcó un hito la Conferencia de las Naciones Unidas sobre Medio Ambiente y Desarrollo en 1992, con sede en Rio de Janeiro, en la que se adoptó la Declaración de Rio. Como refieren Anglés Hernández, M. et $\mathrm{al}^{8}$, aquí se adoptaron dos tratados internacionales de gran relevancia (hard law), la Convención Marco de las Naciones Unidas sobre Cambio Climático (CMNUCC) y el Convenio sobre la Diversidad Biológica (CDB).

En esta Declaración se establecieron 27 principios, entre algunos podemos mencionar:

\begin{tabular}{|l|l|}
\hline (Principio 2 de la Declaración de & "Los Estados tienen el derecho soberano de aprovechar sus \\
Río de 1992 sobre Medio Ambiente & recursos según sus propias políticas ambientales y de \\
y Desarrollo). & $\begin{array}{l}\text { desarrollo y la responsabilidad de velar por que las actividades } \\
\text { realizadas dentro de su jurisdicción y bajo su control no causen } \\
\text { daños al medio ambiente de otros Estados o de zonas que están } \\
\text { fuera de los límites de la jurisdicción nacional". }\end{array}$ \\
\hline
\end{tabular}

7. M. Anglés Hernández, M. Rovalo Otero, M. Tejado Gallegos, Manual de Derecho Ambiental Mexicano, (2021), p. 3. https://archivos.juridicas.unam.mx/www/bjv/libros/13/6429/13.pdf

8. Op. cit, 


\begin{tabular}{|l|l|}
\hline $\begin{array}{l}\text { (Principio 3 de la Declaración de } \\
\text { Rio, 1992). }\end{array}$ & $\begin{array}{l}\text { "El derecho al desarrollo debe ejercerse en forma tal que } \\
\text { responda equitativamente a las necesidades de desarrollo y } \\
\text { ambientales de las generaciones presentes y futuras". }\end{array}$ \\
\hline $\begin{array}{l}\text { (Principio 4 de la Declaración de } \\
\text { Rio, 1992) }\end{array}$ & $\begin{array}{l}\text { "A fin de alcanzar el desarrollo sostenible, la protección del } \\
\text { medio ambiente deber constituir parte integrante del proceso } \\
\text { Re desarrollo y no podrá considerarse en forma aislada". }\end{array}$ \\
\hline Principio 17 de la Declaración de & $\begin{array}{l}\text { "Debe emprenderse una evaluación del impacto ambiental, en } \\
\text { calidad de instrumento nacional, respecto de cualquier } \\
\text { actividad propuesta que probablemente haya de producir un } \\
\text { impacto negativo considerable en el medio ambiente y que esté } \\
\text { sujeta a la decisión de una autoridad nacional competente". }\end{array}$ \\
\hline
\end{tabular}

Tabla 2. Elaboración propia

El Derecho Internacional Ambiental constituye pues, un régimen jurídico autónomo, si bien tiene como base el sistema del derecho internacional público, Drnas de Clément, ${ }^{9}$, Constituye el conjunto de normas jurídicas internacionales que regulan la conducta de los Estados referido a la protección, preservación y conservación del ambiente ${ }^{10}$

\section{CAMBIO CLIMÁTICO}

El cambio climático es definido jurídicamente como aquel cambio de clima atribuido directa o indirectamente a la actividad humana que altera la composición de la atmósfera global y que se suma a la variabilidad natural del clima observada durante períodos de tiempo comparables y diferencia entre el cambio climático atribuible a las actividades humanas que alteran la composición atmosférica y la variabilidad del clima atribuible a causas naturales. (artículo 1 de la Convención Marco sobre Cambio Climático)

El Grupo Intergubernamental de Expertos sobre el Cambio Climático (IPCC, por sus siglas en inglés) fue creado en 1988 y se encarga de realizar evaluaciones integrales del estado de los conocimientos científicos, técnicos y socioeconómicos, sobre las causas, repercusiones del cambio climático y estrategias de respuesta. (IPCC, s. f.)

9. Z. Drnas de Clément, «RECORDIP». Fuentes del Derecho Internacional del Medio Ambiente. (Cordoba: editado por Departamento de Derecho Público de la Facultad de Derecho de la Universidad Nacional de Córdoba. 2009).

https://revistas.unc.edu.ar/index.php/recordip/article/view/37

10. M. Anglés, et al , op. cit., p.1 
Es importante hacer un poco de historia, recordando la XV Conferencia Internacional sobre el Cambio Climático (en siglas COP15). Esta Conferencia internacional celebrada en la ciudad de Copenhague, capital de Dinamarca del 7 al 18 de diciembre de 2009. Esta reunión fue organizada por la Convención Marco de las Naciones Unidas sobre el Cambio Climático (CMNUCC), institución que organiza conferencias anuales desde 1995 con la meta de preparar futuros objetivos pro Cambio Climático.

\section{A: Objetivos}

\begin{tabular}{|l|l|}
\hline $\begin{array}{l}\text { La conclusión de un acuerdo jurídicamente } \\
\text { vinculante sobre el clima. }\end{array}$ & $\begin{array}{l}\text { Válido en todo el mundo, con perspectivas a ser } \\
\text { aplicado a partir de 2012. }\end{array}$ \\
\hline $\begin{array}{l}\text { La reducción mundial de las emisiones de CO2 en al } \\
\text { menos un 50\% en 2050 respecto a 1990, y para }\end{array}$ & $\begin{array}{l}\text { Los países industrializados deberían reducir sus } \\
\text { emisiones de gases de efecto invernadero entre un } \\
\text { conseguirlo los países debían marcarse objetivos } \\
\text { intermedios. }\end{array}$ \\
& $\begin{array}{l}25 \% \text { y un } 40 \% \text {, respecto a los niveles de } 1990 \text { en el } \\
\text { año } 2020 \text { y deberían alcanzar una reducción entre el } \\
80 \% \text { y el 95\% para 2050. }\end{array}$ \\
\hline
\end{tabular}

Tabla 3. Elaboración propia

\section{B: Negociaciones}

\begin{tabular}{|l|l|}
\hline El dilema era cómo promover el crecimiento & El punto de vista de estos países es que la mayor parte \\
económico sin perjudicar el medio ambiente. & del cambio climático está alimentado por la demanda \\
& de los países ricos; por eso consideran que es injusto \\
& penalizarlos por el uso de combustibles fósiles para \\
& fabricar bienes que se consumen en los países ricos. \\
& Sin embargo, ciertos países en desarrollo se \\
& comprometieron también a imponerse objetivos de \\
& reducción ${ }^{11}$.
\end{tabular}

11. En marzo de 2009, México fue el primer país en desarrollo en proponer una meta para reducir sus emisiones. En septiembre de 2009, China anunció un plan en el que propuso reducir la intensidad de sus emisiones. En noviembre de 2009, Brasil propuso reducir las emisiones derivadas de la deforestación, que es la principal fuente de emisiones de gases de efecto invernadero, en un $80 \%$ en 2020 . 


\begin{tabular}{|l|l|}
\hline Cooperación para ayudar a los países pobres & $\begin{array}{l}\text { La ONU consideró que los países desarrollados } \\
\text { deberían ayudar a los países pobres a prepararse para } \\
\text { las consecuencias del cambio climático; por eso creó } \\
\text { un Fondo de Adaptación, un paquete financiero } \\
\text { diseñado para ayudar a los países en desarrollo a } \\
\text { proteger sus economías contra el impacto potencial } \\
\text { del cambio climático }{ }^{12} .\end{array}$ \\
\hline
\end{tabular}

Tabla 4. Elaboración propia

\section{C: Acuerdos}

En la última noche de la cumbre se gestó el acuerdo final entre cuatro grandes países emergentes India, Brasil, Sudáfrica, y EE.UU.

FINANCIACIÓN PARA LAS
NACIONES POBRES

El texto dice: "Las naciones desarrolladas deben aportar recursos financieros adecuados, previsibles, sostenibles y apoyo tecnológico para crear dicha capacidad, para apoyar la implementación de acciones de adaptación en los países en desarrollo".

\section{REDUCCIÓN DE LAS EMISIONES}

Los detalles de los planes de reducción se incluyen en dos anexos separados: uno con objetivos para los países desarrollados y uno para los compromisos voluntarios para la mayoría de los países en desarrollo.
"La delegación india propuso un tratado no vinculante que siguiera el modelo de la Organización Mundial del Comercio donde cada país declarara sus emisiones. El acuerdo no cumplió las expectativas para la cumbre de Copenhague".

Los países desarrollados establecieron un objetivo de movilizar conjuntamente 100,000 millones de dólares al año para 2020 para abordar las necesidades de los países en desarrollo. Los fondos procederán de una serie de fuentes, públicas y privadas, bilaterales y multilaterales.

Estos no son vinculantes y describen el actual estatus de promesas - que oscilan desde el "en consideración" de Estados Unidos a la "legislación adoptada" de la Unión Europea.

12. El problema es que los países desarrollados no deseaban financiar este plan, aunque la Unión Europea anunció un acuerdo para dar 7,200 millones de euros hasta 2012 a los países en vías de desarrollo para que luchen contra el cambio climático. En el caso de España, la ayuda a los países pobres, según anunció el presidente del Gobierno, José Luis Rodríguez Zapatero, será de 375 millones de euros en los tres años. Suecia ha comprometido 800 millones de euros, Italia 600 y Holanda 300 millones de euros. 


\begin{tabular}{|l|l|}
\hline VERIFICACIÓN & $\begin{array}{l}\text { Un punto conflictivo, en gran medida porque China se negaba a } \\
\text { aceptar los controles internacionales, la sección de compromisos } \\
\text { de supervisión sobre las naciones en desarrollo es una de las más } \\
\text { largas del acuerdo. Dice que las economías emergentes deben } \\
\text { vigilar sus esfuerzos y comunicar los resultados a las Naciones } \\
\text { Unidas cada dos años, con algunas comprobaciones } \\
\text { internacionales para comprobar las inquietudes occidentales sobre } \\
\text { la transparencia, pero "para asegurar que la soberanía nacional se } \\
\text { respeta". }\end{array}$ \\
\hline PROTECCIÓN DE LOS BOSQUES & $\begin{array}{l}\text { El acuerdo reconoce la importancia de reducir la emisión de gases } \\
\text { procedentes de la deforestación y la degradación de los bosques y } \\
\text { la necesidad de mejorar la eliminación de los gases de efecto } \\
\text { invernadero que proporcionan los bosques' y acuerda aportar } \\
\text { "incentivos positivos" para financiar tales acciones con recursos } \\
\text { financieros del mundo desarrollado. }\end{array}$ \\
\hline MERCADOS DE CARBONO & $\begin{array}{l}\text { Mencionado, pero no en detalle. El acuerdo dice: "Decidimos } \\
\text { perseguir varias estrategias, incluyendo oportunidades para } \\
\text { utilizar los mercados para mejorar la efectividad-coste y promover } \\
\text { las acciones atenuantes". }\end{array}$ \\
\hline
\end{tabular}

Tabla 5. Elaboración propia

\section{REGULACIÓN INTERNACIONAL DEL CAMBIO CLIMÁTICO}

El desarrollo de la regulación internacional sobre cambio climático lo comprenden tres tratados internacionales:

a) La Convención Marco de las Naciones Unidas sobre Cambio Climático (CMNUCC): Adoptado en 1992

Su objetivo es lograr la estabilización de las concentraciones de gases de efecto invernadero en la atmósfera a un nivel que impida interferencias antropógenas peligrosas en el sistema climático. Ese nivel debería lograrse en un plazo suficiente para permitir que los ecosistemas se adapten naturalmente al cambio climático, asegurar que la producción de alimentos no se vea amenazada y permitir que el desarrollo económico prosiga de manera sostenible" (artículo 2 de la Convención Marco). 


\section{b) Protocolo de Kyoto: Adoptado en 1997}

Su objetivo es que las Partes incluidas en el anexo I se asegurarán, individual o conjuntamente, de que sus emisiones antropógenas agregadas, expresadas en dióxido de carbono equivalente, de los gases de efecto invernadero enumerados en el anexo A no excedan de las cantidades atribuidas a ellas, calculadas en función de los compromisos cuantificados de limitación y reducción de las emisiones consignados para ellas en el anexo B y de conformidad con lo dispuesto en el presente artículo, con miras a reducir el total de sus emisiones de esos gases a un nivel inferior en no menos de 5\% al de 1990 en el período de compromiso comprendido entre el año 2008 y el 2012. (artículo 3.1 del Protocolo).

\section{c) El Acuerdo de Paris: Adoptado en 2015}

$\mathrm{Su}$ objetivo es mantener el aumento de la temperatura media mundial muy por debajo de $2^{\circ} \mathrm{C}$ con respecto a los niveles preindustriales, y proseguir los esfuerzos para limitar ese aumento de la temperatura a $1,5^{\circ} \mathrm{C}$ con respecto a los niveles preindustriales, reconociendo que ello reduciría considerablemente los riesgos y los efectos del cambio climático. (artículo 2.a)

Como se infiere el cumplimiento de compromisos y obligaciones de los Estados en el marco de estos tratados internacionales, sobre de este último, el Acuerdo de Paris, que introduce como deber para los Estados parte, la presentación de sus contribuciones determinadas a nivel nacional (NDC, por sus siglas en inglés), reportar, monitorear e implementarlas, se rige bajo el principio de responsabilidades comunes y diferenciadas de acuerdo a sus capacidades. De otro lado los Estados se ha comprometido conseguir los objetivos de la Agenda 2030, adoptada también en 2015, con 17 Objetivos de Desarrollo Sostenible.

\section{d) Cambio climático y derechos humanos}

El Consejo de Derechos Humanos ha adoptado una serie de resoluciones en las que establece la relación entre los derechos humanos y el cambio climático (ACNUDH, s. f.). Algunas resoluciones son: 


\begin{tabular}{|l|l|}
\hline \multicolumn{1}{|c|}{ Resolución } & \multicolumn{1}{c|}{ Desarrollo } \\
\hline $\begin{array}{l}\text { Resolución 47/24 (julio de } \\
\text { 2021) }\end{array}$ & $\begin{array}{l}\text { El Consejo reconoció que los derechos de las personas en situaciones vulnerables } \\
\text { se vieron afectados de manera desproporcionada por el impacto negativo del } \\
\text { cambio climático. Solicitó al secretario general que preparara un informe sobre } \\
\text { la cuestión y decidió que en la } 50^{\text {a }} \text { reunión del Consejo se celebrara una mesa } \\
\text { redonda sobre el tema }{ }^{13}\end{array}$ \\
\hline $\begin{array}{l}\text { Resolución 44/7 (julio de } \\
\text { 2020) }\end{array}$ & $\begin{array}{l}\text { El Consejo reconoció que los impactos negativos del cambio climático afectaron } \\
\text { de manera desproporcionada los derechos de las personas mayores. Solicitó al } \\
\text { ACNUDH que llevara a cabo un estudio sobre la cuestión y decidió que en la 47 } \\
\text { sesión del Consejo se celebrara una mesa redonda sobre este tema. }\end{array}$ \\
\hline Resolución 42/21 (julio de & $\begin{array}{l}\text { El Consejo reconoció que los derechos de las personas con discapacidad se ven } \\
\text { afectados de manera desproporcionada por los impactos negativos del cambio } \\
\text { climático. Se pidió a la Oficina que prepare un estudio analítico sobre la } \\
\text { promoción y la protección de los derechos de las personas con discapacidad en } \\
\text { el contexto del cambio climático y un panel de discusión en su 44 }{ }^{\circ} \text { período de } \\
\text { sesiones sobre el mismo tema }\end{array}$ \\
\hline $\begin{array}{l}\text { Resolución 38/4 (julio de } \\
2018)\end{array}$ & $\begin{array}{l}\text { El Consejo reconoció que la integración de un enfoque sensible al género en las } \\
\text { políticas climáticas aumentaría la efectividad de la mitigación y adaptación al } \\
\text { cambio climático, solicitando un estudio analítico y una mesa redonda sobre el } \\
\text { tema. }\end{array}$ \\
\hline
\end{tabular}

13. El Consejo también decidió incorporar en su programa de trabajo anual, a partir de 2023, una mesa redonda sobre diferentes temas relacionados con el cambio climático y los derechos humanos. Alentó a que prosiguieran los debates entre los Estados y las partes interesadas pertinentes sobre la posible creación de un nuevo mandato de procedimiento especial sobre el cambio climático y los derechos humanos. 


\begin{tabular}{|c|c|}
\hline $\begin{array}{l}\text { Resolución 35/20 (julio de } \\
\text { 2017) }\end{array}$ & $\begin{array}{l}\text { El Consejo tomó nota de la urgencia de proteger y promover los derechos } \\
\text { humanos de los migrantes y las personas desplazadas a través de las fronteras } \\
\text { internacionales en el contexto del impacto adverso del cambio climático }{ }^{14} \text {. }\end{array}$ \\
\hline $\begin{array}{l}\text { Resolución 32/33 (julio de } \\
\text { 2016) }\end{array}$ & $\begin{array}{l}\text { El Consejo instó a las Partes a integrar los derechos humanos en la mitigación y } \\
\text { adaptación al cambio climático, y pidió que se celebrara una mesa redonda sobre } \\
\text { el impacto adverso del cambio climático en los derechos del niño en su } 34^{\circ} \\
\text { período de sesiones. }\end{array}$ \\
\hline $\begin{array}{l}\text { Resolución 29/15 (julio de } \\
\text { 2015) }\end{array}$ & $\begin{array}{l}\text { El Consejo hizo hincapié en la urgencia de seguir abordando las consecuencias } \\
\text { adversas del cambio climático para todos y pidió una mesa redonda y un estudio } \\
\text { analítico sobre los impactos del cambio climático en el disfrute del derecho a la } \\
\text { salud. }\end{array}$ \\
\hline $\begin{array}{l}\text { Resolución 26/27 (julio de } \\
\text { 2014) }\end{array}$ & $\begin{array}{l}\text { El Consejo hizo hincapié en la necesidad de que todos los Estados mejoren el } \\
\text { diálogo y la cooperación internacionales para abordar los impactos adversos del } \\
\text { cambio climático en el disfrute de los derechos humanos, incluido el derecho al } \\
\text { desarrollo }^{15} \text {. }\end{array}$ \\
\hline $\begin{array}{l}\text { Resolución } \\
\text { 18/22 (septiembre de 2011) }\end{array}$ & $\begin{array}{l}\text { El Consejo afirmó que las obligaciones, las normas y los principios de derechos } \\
\text { humanos tienen el potencial de informar y fortalecer la formulación de políticas } \\
\text { nacionales e internacionales en el ámbito del cambio climático, promoviendo la } \\
\text { coherencia, la legitimidad y los resultados sostenibles de las políticas. }\end{array}$ \\
\hline $\begin{array}{l}\text { Resolución 10/4 (marzo de } \\
\text { 2009) }\end{array}$ & $\begin{array}{l}\text { El Consejo señaló que "los impactos relacionados con el cambio climático tienen } \\
\text { una variedad de implicaciones, tanto directas como indirectas, para el goce } \\
\text { efectivo de los derechos humanos ..." y que esos efectos "se sentirán más } \\
\text { agudamente por aquellos segmentos de la población que ya se encuentran en } \\
\text { situación de vulnerabilidad...". }\end{array}$ \\
\hline $\begin{array}{l}\text { Resolución 7/23 (marzo de } \\
\text { 2008) }\end{array}$ & $\begin{array}{l}\text { El Consejo expresó su preocupación de que el cambio climático "representa una } \\
\text { amenaza inmediata y de gran alcance para las personas y las comunidades de } \\
\text { todo el mundo" y solicitó al ACNUDH que preparara un estudio sobre la relación } \\
\text { entre el cambio climático y los derechos humanos. }\end{array}$ \\
\hline
\end{tabular}

Tabla 6. Elaboración propia.

14. Pidió una mesa redonda entre sesiones sobre el tema, así como un informe sobre las brechas en la protección de los derechos humanos en el contexto de la migración y el desplazamiento de personas a través de las fronteras internacionales.

15. Hizo un llamado al diálogo, la creación de capacidad, la movilización de recursos financieros, la transferencia de tecnología y otras formas de cooperación para facilitar la adaptación y mitigación del cambio climático, a fin de satisfacer las necesidades y circunstancias especiales de los países en desarrollo. 
Por último, actualmente el Consejo de Derechos Humanos adoptó en 2021 la Resolución 48/13, la cual reconoce por primera vez que tener un medio ambiente limpio, saludable y sostenible es un derecho humano. (ACNUDH, 2021)

\section{e) Jurisprudencia: El Caso Urgenda contra Países Bajos y el cambio climático}

A nivel de pronunciamiento de cortes internacionales y naciones sobre la materia, es importante mencionar que existen pronunciamientos judiciales que tomaron como referencia la afectación a derechos humanos y el cumplimiento de los compromisos internacionales climáticos. Un ejemplo de ello es, en el 2012, la Fundación Urgenda demandó al gobierno de Países Bajos ante el Tribunal de la Haya, solicitando que el Estado limite el volumen de las emisiones anuales de gases de efecto invernadero para que estas emisiones se hayan reducido en un $40 \%$ y al menos en un $25 \%$ en 2020 en comparación con 1990.

En primera instancia: El 2015 el Tribunal de ese país ordenó al gobierno reducir sus emisiones en un $25 \%$ en 2020 respecto a los niveles de 1990, basándose en la CMNUCC y el Convenio Europeo para la Protección de los Derechos Humanos y de las Libertades Fundamentales (CEDH), argumentado el derecho a la vida (artículo 2) y el derecho a la vida privada y familiar, así como a la inviolabilidad del domicilio (artículo 8).

En segunda instancia: El Tribunal de Apelación mediante sentencia ECLI: NL: GHDHA: 2018: 2610, confirmó que el Estado contravino los artículos 2 y 8 del CEDH y ordenó al gobierno que tome medidas para reducir sus emisiones en un 25\% para fines de 2020.

El caso antes mencionado forma parte de los litigios climáticos que se están desarrollando. Según el Informe Mundial sobre litigios climáticos Revisión Global 2020, al 1 de julio de 2020, al menos 1.550 casos de cambio climático se presentaron en 38 países, lo que está impulsando una evolución necesaria. (PNUMA, 2021). Los litigios sobre cambio climático están cobrando mayor relevancia en las cortes nacionales e internacionales, en donde buscan responsabilizar a los gobiernos y actores corporativos por el incumplimiento de tratados internacionales como la Convención Marco de las Naciones Unidas sobre Cambio Climático, el Acuerdo de París y los compromisos de la Agenda 2030. Esto representa un desafío para el derecho ambiental, pues la atención de las demandas planteadas requiere de la innovación jurídica en materia de cambio climático y derechos humanos. ${ }^{16}$

\section{CONCLUSIONES Y RECOMENDACIONES}

El derecho internacional ambiental tiene una evolución desde hace más de cuatro décadas, tiene como origen la Declaración de Estocolmo de 1972 y comprende el conjunto de normas internacionales

16. L. Orosco López, "Litigio climático: avances en las practicas de Cortes nacionales de los Estados". Taller de Derecho Ambiental. 2021.

http://tdaunmsm.com/2021/10/01/litigios-climaticos-avances-en-las-practicas-de-cortes-nacionales-de-los-estados/ 
que regulan las conductas de Estados sobre la protección y preservación del ambiente. En ese sentido, el cambio climático es atribuido directa o indirectamente a la actividad humana que altera la composición de la atmósfera global. La ciencia especializada señala que urge una acción trascendental.

La regulación del cambio climático, expresada en tratados internacionales que comprenden la Convención Marco de las Naciones Unidas sobre Cambio Climático, el Protocolo de Kyoto y el Acuerdo de Paris, que tienen como objetivo limitar las emisiones de gases de efecto invernadero y contrarrestar los efectos del cambio climático y protección del medio ambiente. A pesar de que los Estados han tratado de implementar dichas obligaciones y compromisos, vemos que no se están cumpliendo de manera ambiciosa y vemos los impactos del cambio climático a los derechos humanos que se agudizan para la población en condición vulnerable.

Está en curso su compromiso político en alcanzar la Agenda 2030 sobre desarrollo sostenible. A nivel de pronunciamiento internacional se debe destacar las resoluciones del Consejos de Derecho Humanos sobre cambio climático y derechos humanos. A nivel de cortes judiciales sobre cambio climático, vemos como los denominados litigios climáticos en cortes nacionales, están yendo en aumento, justamente alegan afectación a derechos humanos y de tratados internacionales, como lo es el caso Urgenda contra el gobierno de Países Bajos. Estos casos seguirán en aumento y se espera que a ello se sumen también los pronunciamientos de cortes internacionales. Veremos más adelante el desarrollo sobre esta materia y compelir a que los Estados cumplan efectivamente dichas obligaciones y compromisos.

\section{REFERENCIAS}

- ACNUDH. Bachelet elogia histórico reconocimiento del derecho a un ambiente saludable.

08 de octubre de 2021.

http://www.oacnudh.org/bachelet-elogia-historico-reconocimiento-del-derecho-a-unambiente-saludable/

- Resoluciones del Consejo de Derechos Humanos sobre derechos humanos y cambio climático. s.f. https://www.ohchr.org/EN/Issues/HRAndClimateChange/Pages/Resolutions.aspx

- Acuerdo de Paris. 2015. https://unfccc.int/sites/default/files/spanish_paris_agreement.pdf

- $\quad$ Anglés Hernández, M., Rovalo Otero, M., Tejado Gallegos. M. Manual de Derecho Ambiental Mexicano, 2021. https://archivos.juridicas.unam.mx/www/bjv/libros/13/6429/13.pdf

- Carta de la Organización de Estados Americanos. 1948. http://www.oas.org/es/sla/ddi/tratados_multilaterales_interamericanos_A-41_carta_ OEA.asp 
- Carta Mundial de la Naturaleza. 1982.

https://www.iri.edu.ar/publicaciones_iri/manual/UltimaTanda/Medio\%20Ambiente/7.\%

20CartaMundialdelaNaturaleza.pdf

- Convención Americana sobre Derechos Humanos. 1969.

https://www.corteidh.or.cr/tablas/17229a.pdf

- Convención Marco de las Naciones Unidas sobre el Cambio Climático. 1992. https://unfccc.int/files/essential_background/background_publications_htmlpdf/ application/pdf/convsp.pdf

- Cumbre Mundial sobre el Desarrollo Sostenible (Johannesburgo). 2002. https://www.un.org/spanish/esa/sustdev/WSSDsp_PD.htm

- Cumbre Social. 1995. https://www.acnur.org/fileadmin/Documentos/BDL/2016/10701.pdf

- "Declaración Americana de Derechos y Deberes del Hombre”. 1948. http://www.oas.org/es/cidh/mandato/Basicos/declaracion.asp

- "Declaración de Estocolmo para la Preservación y Mejoramiento del Medio Humano". Conferencia de las Naciones Unidas sobre el Medio Humano, Estocolmo, 1972. http://www.ordenjuridico.gob.mx/TratInt/Derechos\%20Humanos/INST\%2005.pdf

- "Declaración de la Conferencia de las Naciones Unidas sobre el Medio Ambiente y Desarrollo”. 1992. https:/www.un.org/spanish/esa/sustdev/agenda21/riodeclaration.htm

- "Declaración de principios y recomendaciones para la acción de la Conferencia de las Naciones Unidas sobre el Medio Humano". 1972. http://www.ordenjuridico.gob.mx/TratInt/Derechos\%20Humanos/INST\%2005.pdf

- "Declaración de Río de Janeiro sobre Medio Ambiente y Desarrollo de la Conferencia de las Naciones Unidas sobre el Medio Ambiente y Desarrollo, Río de Janeiro". 1992. https://www.un.org/spanish/esa/sustdev/agenda21/riodeclaration.htm

- "Declaración de Viena”. 1993. https://www.ohchr.org/sp/newsevents/ohchr20/pages/wchr.aspx .

- “Declaración del Milenio de 2000 y los Objetivos de Desarrollo del Milenio”. 2000. https://undocs.org/es/A/RES/55/2 
- "Declaración sobre el Desarrollo". 1987. https://www.un.org/es/ga/president/65/issues/sustdev.shtml

- "Declaración Universal de Derechos Humanos". 1948. https://www.un.org/es/about-us/universal-declaration-of-human-rights

- $\quad$ Drnas de Clément, Z. "RECORDIP”. Fuentes del Derecho Internacional del Medio Ambiente. Cordoba: editado por Departamento de Derecho Público de la Facultad de Derecho de la Universidad Nacional de Córdoba. 2009. https://revistas.unc.edu.ar/index.php/recordip/article/view/37

- “IPCC”. actividades. s.f. https://archive.ipcc.ch/home_languages_main_spanish.shtml

- Orosco López, L. "Litigio climático: avances en las prácticas de Cortes nacionales de los Estados". Taller de Derecho Ambiental. 2021.

http://tdaunmsm.com/2021/10/01/litigios-climaticos-avances-en-las-practicas-de-cortesnacionales-de-los-estados/

- " "Pacto Internacional de Derechos Civiles y Político". 1966. https://www.ohchr.org/sp/professionalinterest/pages/ccpr.aspx

- $\quad$ "Pacto Internacional de Derechos Económicos, Sociales y Cultural". 1966. https://www.corteidh.or.cr/tablas/28142.pdf

- "PNUMA". Informe mundial sobre litigios climáticos. Revisión Global 2020, 26 de enero de 2021. https://www.unep.org/es/resources/informe/informe-mundial-sobre-litigiosclimaticos-revision-global-2020

- $\quad$ "Protocolo de Kyoto", 1997. https://www.congreso.gob.pe/Docs/ParlamentoAmazonico/files /tratados/protocolo-kyoto-convencion.pdf

- "Resolución 10/4", marzo de 2009. https://ap.ohchr.org/documents/E/HRC/resolutions/ A_HRC_RES_10_4.pdf

- “Resolución 18/22”, septiembre de 2011. https://undocs.org/A/HRC/RES/18/22

- “Resolución 26/27”, julio de 2014. https://undocs.org/A/HRC/RES/26/27

- "Resolución 29/15”, julio de 2015. https://undocs.org/A/HRC/RES/29/15

- "Resolución 32/33”, julio de 2016. https://undocs.org/A/HRC/RES/32/33

- "Resolución 35/20”, julio de 2017. https://undocs.org/A/HRC/RES/35/20 
- "Resolución 38/4”, julio de 2018. https://undocs.org/A/HRC/RES/38/4

- “Resolución 42/21”, julio de 2019. https://undocs.org/A/HRC/RES/41/21

- "Resolución 44/7", julio de 2021. https://undocs.org/A/HRC/RES/44/7

- “Resolución 47/24”, julio de 2021. https://undocs.org/A/HRC/RES/47/24

- "Resolución 7/23", marzo de 2008.

https://ap.ohchr.org/documents/E/HRC/resolutions/A_HRC_RES_7_23.pdf

- "Sentencia contra Países Bajos". ECLI:NL:GHDHA:2018:2610. Tribunal de Apelación. 2018. http://blogs2.law.columbia.edu/climatechangelitigation/wpcontent/uploads/sites/16 /non-us-casedocuments/2018/20181009_2015-HAZA-C0900456689_decision-4.pdf 